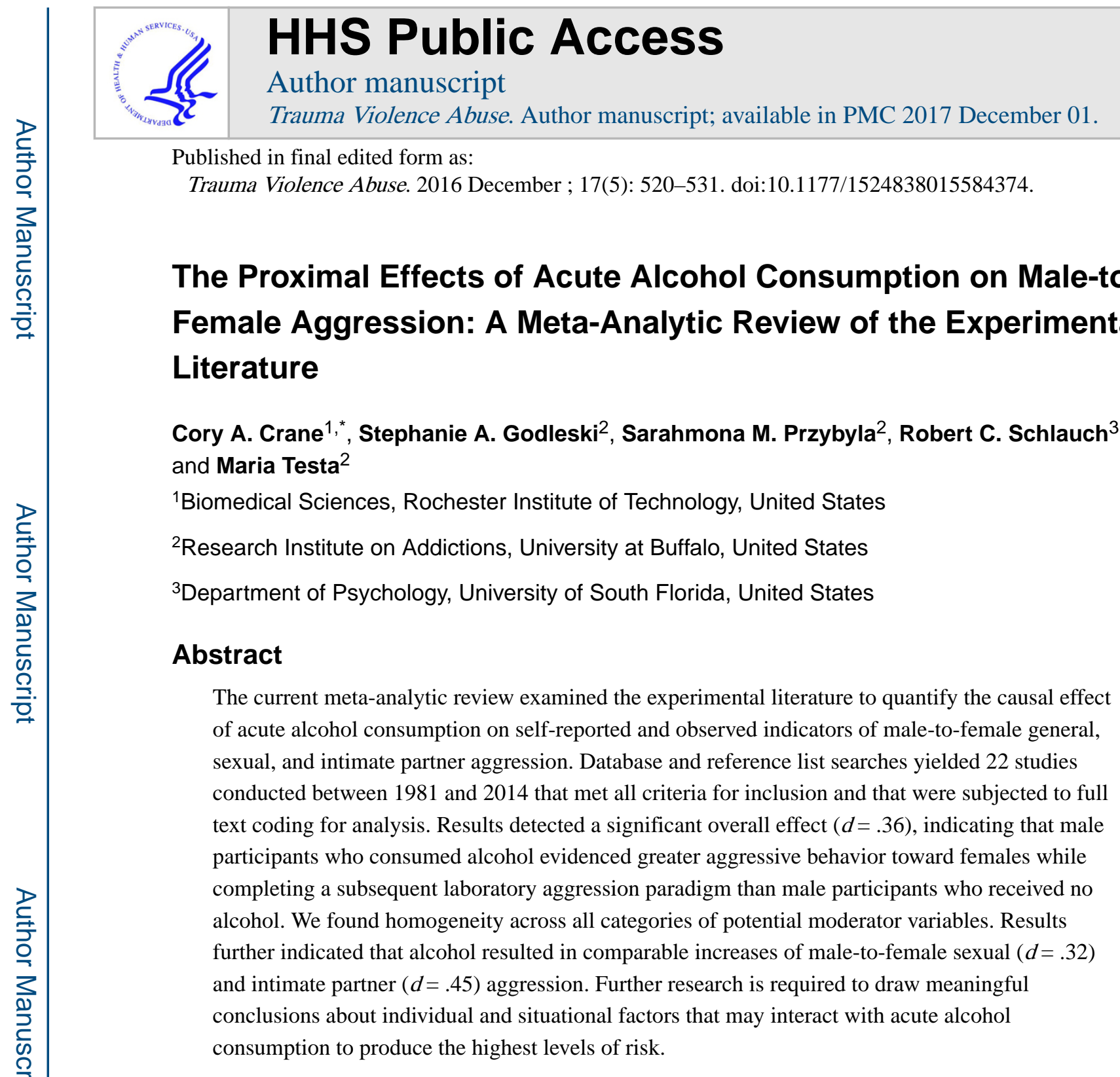

\title{
Keywords
}

Alcohol; Sexual Aggression; Intimate Partner Aggression; Meta-Analysis; Experiment

\begin{abstract}
Male-to-female aggression remains a significant public health concern. Although approached as disparate areas, an examination of the existing sexual aggression (SA) and intimate partner aggression (IPA) literatures reveals considerable commonalities between these two types of male-to-female aggression. SA refers to any forceful or coercive behavior used to engage in sexual activity with an otherwise unwilling or incapacitated partner (e.g., Abbey et al., in press). IPA encompasses a wide variety of aggressive behavior, including physical violence, directed toward a current or past relationship partner (e.g., Saltzman et al., 2002). An examination of male-to-female aggression is warranted by both high prevalence as well as the severity of associated consequences. Previous meta-analytic findings indicate
\end{abstract}

*Corresponding author: Cory A. Crane, Rochester Institute of Technology, Biomedical Sciences, 153 Lomb Memorial Drive, Rochester, NY 14623, Phone: (585) 475-4018, Fax: (585) 475-4360, cacihst@ rit.edu. 
that males may be more likely to perpetrate physical $(d=.39-.84)$ and verbal $(d=.14-.51)$ aggression than females (Archer, 2004). In considering inter-gender aggression, sexual dimorphism suggests that aggressive males may be capable of inflicting greater harm than aggressive females. Indeed, even under conditions in which females perpetrate more frequent aggression than males, severe aggression involving serious injury or hospitalization is disproportionately associated with male perpetration (Archer, 2000). Nationally representative epidemiological data collected through the 2011 National Intimate Partner and Sexual Violence Survey of all non-institutionalized English and Spanish speaking adults in the United States indicate that 19.3\% of females have been victims of rape, $43.9 \%$ endured other sexual victimization, and $31.5 \%$ reported experiencing physical IPA in their lifetimes (Breiding et al., 2014). Thus, victimization qualifies for inclusion among the leading causes of preventable morbidity and mortality among females in the United States and abroad (Centers for Disease Control and Prevention, 2014). Beyond immediate distress, SA and IPA have also been associated with myriad adverse, long-term mental and physical health consequences among female victims (e.g., Coker, et al., 2002).

\section{Alcohol and Aggression}

Given the gravity of this significant public health concern, researchers have attempted to detect proximal factors that most reliably predict aggressive outcomes. Initial survey research provided a preponderance of evidence to support distal associations between alcohol use characteristics and reports of aggressive behavior (e.g., Abbey \& Jacques-Tiura, 2011; Foran \& O'Leary, 2008). Event-based research supports a proximal relationship between alcohol and aggression (e.g., Testa \& Derrick, 2014), suggesting that alcohol may be involved in upwards of $50 \%$ of male-to-female aggressive episodes, including homicides as well as physically and sexually aggressive offenses (e.g., Perkins, 2002; Roizen, 1997). Taken together, these methods indicate that individuals who consume greater amounts of alcohol also evidence increased rates of IPA and SA perpetration in general and that acute alcohol use may be associated with an increased risk of aggression during specific interactions.

Unlike cross-sectional and naturalistic designs, which introduce potential situational and dispositional confounds, experimental methodologies are ideally suited to assess the direct, proximal effects of acute alcohol consumption on male-to-female aggression under carefully controlled conditions. Laboratory analog procedures are unique in their ability to provide evidence of a causal relationship between acute alcohol use and an increased risk of perpetrating SA (e.g., Abbey, McAuslan, \& Ross, 1998) as well as IPA (e.g., Eckhardt, 2007). The current review, however, is the first to comprehensively evaluate experimental analog studies of male-to-female aggression following alcohol consumption and to quantify the potentially distinct effect of alcohol on SA relative to IPA.

Proximal effects models are among the most compelling and well supported theoretical explanations of the robust relationship observed between acute alcohol use and aggression. These models focus on the direct psychopharmacological effects of alcohol which depress higher order executive functioning that aids in the inhibition of normative aggressive impulses, efficient social information processing, and decision making (Giancola, Josephs, 
Parrott, \& Duke, 2010). Contemporary models of alcohol myopia and disinhibition posit that alcohol serves to instigate aggression through a restriction of cognitive resources to only the most salient environmental cues at the sacrifice of less salient or long-term inhibitory influences (Steele \& Josephs, 1990; Taylor \& Leonard, 1983). For example, with the SA paradigm, alcohol is thought to increase focus on the dominant cues consistent with sexual arousal while impairing the ability to focus on the less salient, inhibitory cues (e.g., commitment to another relationship or female resistance). Consequently, alcohol reduces inhibitions against acting upon sexual impulses (e.g., Abbey et al., 1998). Applied to IPA, alcohol is thought to increase focus on dominant cues (e.g., insult or anger) related to threat or confrontation. Non-dominant, inhibitory cues (e.g., empathy or affection toward one's partner) are less salient under the influence of alcohol, thereby decreasing the capacity to effect nonaggressive conflict resolution techniques (Clements \& Schumacher, 2010).

\section{Previous Reviews of Alcohol and Aggression}

A substantial body of research offers support for these proximal models depicting the effects of alcohol on male-to-female aggression and has served as the basis of several reviews. Our treatment of these reviews will reflect the distinction imposed between general, sexual, and intimate partner aggression.

\section{General Aggression}

Exum (2006) summarized seven meta-analytic reviews of the relationship between experimentally manipulated alcohol and laboratory aggression, offering consistent evidence to support small to medium sized effects of alcohol on aggression. Only three of the metaanalytic reviews included in Exum's review presented overall effect sizes. Ito and colleagues (1996) assessed the effects presented in 47 studies $(d=.47)$, Bushman (1997) derived a medium overall effect size $(d=.50)$ from 60 studies, and Lipsey and colleagues (1997) summarized data from 42 experimental studies $(d=.54)$. However, each of these reviews included a disproportionate number of studies that evaluated male-to-male, rather than maleto-female, aggression. With generally greater aggression directed toward male than female victims in the absence of alcohol (Eagly \& Steffen, 1986), it is likely that effects reported in generalized aggression meta analyses will poorly represent the effects of alcohol on male-tofemale aggression.

\section{Sexual Aggression}

There have been two qualitative and no quantitative reviews of the effects of alcohol on men's sexual aggression. Testa (2002) reviewed the effects of alcohol on SA using the accumulated associational, event-level, and experimental studies, finding that studies using all three methods provided at least partial support for alcohol-related SA and that experimental analog studies were consistent with sexual disinhibition as a result of the proximal, psychopharmacological effect of alcohol, as indicated by greater SA among participants randomly assigned to an alcohol, rather than a control, condition across investigations. Abbey and colleagues (in press) recently updated a previous review (Abbey, 2011) of survey and experimental research on the effects of alcohol on SA perpetration among males. Contained within the systematic review was a description of 12 experimental 
studies utilizing both aggression paradigms and alcohol manipulation. Findings were consistent with Testa (2002) in identifying alcohol use as a proximal risk factor for male-tofemale SA. These previous reviews describe experimental studies and, thus, are able to comment on a causal relationship between alcohol and SA but we have no composite estimate of the effect because all stopped short of conducting a meta-analysis to quantify this relationship.

\section{Intimate Partner Aggression}

Although previous reviews of survey studies have established a positive association between alcohol consumption and perpetration of IPA, these reviews do not consider the proximal effects of alcohol. Reviews of alcohol-involved IPA have primarily utilized cross sectional, self-report data and find homogeneous effects in the small to medium range. Ferrer and colleagues (2004) found evidence of greater alcohol problems among male IPA perpetrators than nonviolent males across 9 studies $(d=.57)$. Stith and colleagues (2004) analyzed findings from 22 studies to similarly report greater alcohol problems among male IPA perpetrators relative to nonviolent males $(d=.48)$. Most recently, Foran and O'Leary (2008) conducted a more comprehensive review of 47 studies to yield a nearly identical overall effect size $(d=.47)$. Thus, previous reviews evidence consistently moderate effect sizes between alcohol and IPA but are becoming dated and rely almost exclusively upon nonexperimental methodologies with Foran and O'Leary (2008) intentionally excluding laboratory analog studies. As a result, these reviews offer no evidence toward causality, are representative of only the associations between problematic drinking behavior and partner aggression within a specified reference period, and do not assess the proximal effects of acute alcohol consumption on IPA.

Thus, reviews have described but not quantified the proximal relationship between acute alcohol consumption and SA, nor have they presented data to comment upon the potential causal effects of alcohol on IPA as observed through experimental methods. We aim to address these issues in the current review and to assess the relative effects of alcohol on SA in comparison to IPA. We also aim to explore additional potential moderators of the overall alcohol-aggression effect.

\section{The Current Study}

We aimed to identify and review the entire experimental literature on the relationship between acute alcohol consumption and male-to-female aggression in order to synthesize an overall effect that would represent this relationship. Specification of the experimental literature restricts our sample of studies to only the most rigorously controlled and ensures that the overall statistic will reflect a proximal, causal effect of alcohol on male-to-female aggression. We hypothesized that the overall effect would be positive and in the small-tomedium range, indicating that male participants who had consumed alcohol generally exhibited greater aggression toward female targets than sober participants across studies.

We also evaluated six potential moderators of the observed effect sizes. Alcohol research within the domains of SA and IPA has remained distinct yet no quantitative reviews on experimental data have been published in either field, likely due to the limited amount of 
existing research in the independent areas. An examination of the literature suggests smallto-medium effects in both SA and IPA domains (e.g., Foran \& O'Leary, 2008; Testa, 2002), indicating that acute alcohol use may represent a comparable proximal risk factor for both forms of aggression but that it is neither necessary nor sufficient to fully account for the occurrence of either. Thus, we evaluated whether the type of aggressive outcome moderated the relationship between alcohol and aggression. Based upon previous research, we anticipated comparable effect sizes among SA and IPA studies. We also considered whether various characteristics of the included studies moderated the strength of the relationship between alcohol and male-to-female aggression. These potential moderators included: the interactive nature of the aggression paradigm, the assessment method, the sample type, and the level of intoxication. We advanced no a priori hypotheses about these moderators due to a lack of guidance from previous research. Further, we anticipated the possible emergence of meaningful trends in moderator analyses but also that the small size of the specified literature would likely preclude the possibility of establishing significant differences between the categories of our moderators.

\section{Method}

\section{Sample}

We conducted database searches for all research articles describing the effects of an alcohol manipulation on male-to-female aggression that appeared prior to May, 2014. Search parameters involved combinations of keywords, including sets of aggression (i.e., Sexual Aggression, Sexual Assault, Sexual Violence, Partner Aggression, Partner Violence, Domestic Violence, Marital Violence, Dating Violence) and laboratory (i.e., Challenge, Manipulation, Placebo, BAC, BAL, BlAC, BrAC) which were further refined by the terms "alcohol" and either "female" or "women." Our search terms yielded a total of 336 items from PsycINFO and 566 matches from PubMed. Additional studies of potential relevance were identified for review through the references included in SA and IPA review articles. Published and unpublished studies appearing in dissertations were evaluated. An initial review of titles and abstracts resulted in 41 potential studies identified for full text review. Inclusion criteria further reduced the set to 15 peer reviewed publications and three dissertations after full review. First authors were contacted to provide additional information where appropriate, resulting in the inclusion of four initially excluded effects and a total of 22 studies (see Figure).

\section{Procedure}

Inclusion Criteria-Eligible articles were required to meet several stringent criteria to be included in the current review, including: 1) The study needed to present data pertaining to a laboratory based experiment, 2) An alcohol exposed group and a suitable control group (e.g., no alcohol or placebo) needed to be distinct and identifiable, 3) A measure of aggression needed to be present and quantifiable, 4) Studies needed to explicitly specify that all participants or a distinct subgroup of participants were male and that targets of aggression were female, 5) Only the earliest published study that provided sufficient effect size data was retained when duplicate samples were suspected across articles, 6) Sufficient data were required to calculate an effect size for the relationship between alcohol and aggression, and 
7) Papers had to be presented in English. Three Unpublished dissertations that met the inclusion criteria were included in the current sample of 22 studies.

Coding - With the exception of the four studies for which authors provided additional data, all eligible studies $(81.8 \%)$ were fully double coded by the first and second author. Using a structured codebook, both coders recorded information pertaining to study methods, including sample characteristics (e.g., size, age, ethnic composition), measures of aggression, and the alcohol manipulation. Coders also reported the aggression paradigm, interactive nature of the paradigm, the method of quantifying the outcome, the participant's relationship to the victim, and aggression scores. Finally, coders recorded the active alcohol dose and the specified type of control group. Coding disagreements were identified and resolved by discussion or arbitration via the fourth author, resulting in full agreement between coders.

\section{Measures of Aggression}

The vignette is the most common experimental paradigm for evaluating male-to-female SA (e.g., Abbey et al., in press). Twelve of the current included studies, all evaluating sexual aggression, utilized a variant of the vignette paradigm. Vignettes may be presented in written $(\mathrm{k}=7)$, video $(\mathrm{k}=3)$, or audio $(\mathrm{k}=2)$ formats and depict scenarios involving male-tofemale sexual aggression or rape. Participants receive the vignette in either second or third person and outcomes are typically assessed through self-reported intentions to engage in sexual aggression, willingness to force sex, ratings of how inappropriate the male's depicted behavior was, or the lag in time that it takes the participant to identify a behavior as sexually aggressive.

Two SA studies, both dissertations, utilized a form of the sexual imposition paradigm (Miller, 2013; Quinones, 1998). Male participants selected between exposing a disinterested female confederate to sexually explicit or nonsexual images or videos. Image selection, the number of exposures, as well as the length of exposure represented measures of sexual aggression in these studies.

Included IPA studies utilized only two aggression paradigms. Three investigations assessed aggression with a couples' conflict resolution paradigm in which couples identified and discussed topics of disagreement within their relationship (Heyman, Weiss, \& Eddy, 1995). Interactions were recorded and verbal behavior was coded for negativity and hostility. Three additional investigations used the Articulated Thoughts in Simulated Situations paradigm (Davison, Robins, \& Johnson, 1983) in which participants listened to audio recordings of a simulated female intimate partner engaging in ambiguous but potentially provocative interactions with other individuals. Participants verbally reported their thoughts and feelings about the interactions, which were recorded and coded for aggressive content.

Two additional investigations assessed general, rather than sexual or intimate partner, aggression. General male-to-female aggression research is differentiated from SA and IPA studies in that participant aggression is directed toward an unfamiliar female with whom the participant has no social obligation or vested interest. Richardson (1981) assessed aggression using the magnitude of shocks set for a female confederate in a competitive reaction time 
task (Taylor, 1967). Finally, Norlander, Nordmarker, and Archer (1998) used panels of professionals to assess the amount of aggression exhibited by males when asked to draw on an aggression eliciting picture in which a female appeared.

\section{Moderators}

Studies were categorized as using an interactive paradigm if participants interacted with or believed that they had interacted with a genuine female participant (e.g., competitive reaction time task; couples interaction task) rather than a simulated female with whom they had not directly interacted (e.g., written SA vignette; simulated situations paradigm). Similarly, active aggression studies included those in which the participant himself directed aggression toward a real or simulated female (e.g., second person SA vignettes) while passive aggression studies included those in which the participant rated or reacted to the aggression displayed by other males (e.g., third person SA vignettes). Studies were further categorized by assessment method as either self-reported (e.g., vignette paradigms) or observed (e.g., couples' conflict resolution paradigm) aggression, as either a college or community sample, and as high (BAC 2.08) or low (BAC <.08) targeted alcohol dose.

\section{Calculation of Effect Size Estimates}

Means and standard deviations $(\mathrm{k}=13)$, regression coefficients $(\mathrm{k}=3)$, F-values $(\mathrm{k}=3)$, point-biserial correlations $(\mathrm{k}=2)$, and proportions $(\mathrm{k}=1)$ were collected from eligible studies and used to calculate standardized mean differences, Cohen's $d$, between alcohol and control groups. Calculations were made using the online practical meta-analysis effect size calculator (a companion to Lipsey \& Wilson, 2001). In the current analysis, larger positive $d$ values indicate that participants who received alcohol exhibited greater aggression than control participants.

Effects based upon regression coefficients (Abbey, Parkhill, Jacques-Tiura, \& Saenz, 2009; Davis, Norris, George, Martel, \& Heiman, 2006; Norris, Davis, George, Martell, \& Heiman, 2002) and F-values (Leonard \& Roberts, 1998; Martell, 2003; Richardson, 1981) were contingent upon additional variables represented in analytical models. Equivalent group sizes were estimated for studies that failed to report exact group sizes but specified assignment through balanced placebo $(\mathrm{k}=6)$, yoking $(\mathrm{k}=2)$ or randomization to condition $(\mathrm{k}=1)$. Assuming equivalent group sizes provides a conservative estimate of the effect size and exploration of reasonable group size variation within these studies resulted in only modest increases in the effect size estimate. In order to avoid violating the assumption of independence, group means and standard deviations were pooled to produce only one aggregate effect from studies involving more than one control or alcohol group (e.g., balanced placebo or low and high alcohol dose designs). When multiple measures were presented, the most representative proxy measure of direct, physical or sexual aggression was retained for effect size coding (e.g., physical aggressive intentions rather than verbal aggression in response to simulated situations).

In calculating the overall effect size, individual effect sizes were weighted by the inverse of their variance, as suggested by Hedges and Olkin (1985), such that effects derived from larger samples exerted greater influence over the overall effect than those derived from small 
samples. Weighted effect sizes were then used to compute the overall effect and evaluate homogeneity across studies with the SPSS Macro presented by Lipsey and Wilson (2001). Mixed effects models, as a conservative method, were then used to examine potential moderators of the alcohol-aggression relationship that might account for any observed heterogeneity across studies.

\section{Results}

\section{Sample Description}

Twenty-two effect sizes were drawn from the current sample of 19 peer reviewed articles and three dissertations published between 1981 and March, 2014. A total of 2,566 participants are represented across all studies that ranged in sample size from 14 to $334(\mathrm{M}=$ 116.6, $\mathrm{SD}=69.6)$. The majority of effects $(63.6 \%)$ represented SA research, while fewer IPA (27.3\%) and general male-to-female aggression (9.1\%) effects were identified. Most studies assessed aggression among participants who did not directly interact with their targets $(72.7 \%)$. Studies further required participants to actively (63.6\%) or passively (36.4\%) aggress. Of the 22 included studies, 10 (45.5\%) involved observed aggression while $12(54.5 \%)$ involved self-reported aggression, $11(50 \%)$ involved primarily college samples while $8(36.4 \%)$ involved primarily community samples, and $11(50 \%)$ targeted a high alcohol dose while 8 (36.4\%) targeted a lower average alcohol dose. There was little racial diversity with most studies specifying predominantly Caucasian samples (68.2\%), one (4.5\%) identifying primarily African American participants, and $6(27.3 \%)$ that failed to specify a majority. The average age of participants across investigations ranged from 22.3 to 32.3 years. The distribution of individual effect sizes is presented in Table 1.

\section{Overall Effect Size}

A random effects analysis using all eligible studies, each of which produced a single effect size $(\mathrm{k}=22)$, was employed to calculate an overall effect size describing the relationship between acute alcohol consumption and male-to-female aggression (Table 2). The magnitude of the overall effect size was small but significant $(d=.36, p<.001,95 \% \mathrm{CI}=$. 25-.46). Although unpublished studies were included in the current analysis, publication biases against undetectable null findings prompted us to calculate a failsafe N (Orwin, 1983; Rosenthal, 1991). We determined that an additional 20 studies with an effect size of zero would be required to reduce the current overall effect below the suggested cutoff of $d=.20$ for a small effect. Thus, a roughly equal number of unpublished studies would need to find that intoxicated and sober participants exhibited comparable amounts of aggression to reduce the current effect to non-significance.

The magnitude of included effect sizes ranged from -.04 to .78, with larger effect sizes indicating greater aggression among alcohol relative to control participants. An analysis of homogeneity revealed significant variability across effect sizes $(Q(21)=33.64, p=.04)$, suggesting that additional variables may account for variability in the current sampling of effect sizes. Although all effects fell within two standard deviations of the mean effect size, we recalculated the overall effect after removing the two most extreme values $(d=-.04$, Abbey et al., 2009; $d=.78$, Samp \& Monahan, 2009). The adjusted effect size evidenced 
change in neither direction nor magnitude $(d=.36)$. Heterogeneity was slightly reduced but remained marginally significant $(Q(19)=29.30, p=.06)$. Due to the absence of outliers and in order to report the most comprehensive estimate of the relationship between alcohol and male-to-female aggression, all 22 effects were retained for subsequent analyses.

\section{Moderator Analyses}

Type of Aggression-Effect sizes reflected the relationship between alcohol and SA ( $d$ $=.32, k=14, p<.001,95 \% \mathrm{CI}=.19-.44)$, IPA $(d=.45, k=6, p<.001,95 \% \mathrm{CI}=.24-.66)$, and general male-to-female aggression $(d=.46, k=2, p=.11,95 \% \mathrm{CI}=-.11-1.03)$. Dropping general aggression investigations, we found no evidence of significant heterogeneity between SA and IPA effect sizes $\left(Q_{b}(1)=1.05, p=.31\right)$, suggesting that the effect of alcohol on aggression was robust across SA and IPA studies even though the overall IPA effect size approached a medium magnitude while the SA effect size was small. We also found no evidence of variability within SA $\left(Q_{W}(13)=13.20, p=.43\right)$ or IPA $\left(Q_{W}(5)=4.10, p\right.$ $=.54$ ) effect sizes. Results of all moderator analyses are displayed in Table 2.

Interaction-An analysis of homogeneity $\left(Q_{b}(1)=.01, p=.90\right)$ revealed that effect sizes derived from procedures in which participants interacted with the target of their aggression $(d=.35, k=6, p<.01,95 \% \mathrm{CI}=.13-.56)$ did not differ from effect sizes in which participants were disconnected from the target of their aggression $(d=.36, \mathrm{k}=16, p<.001$, $95 \% \mathrm{CI}=.24-.48)$. Non-significant variance was detected within groups of interactive $\left(Q_{W}(5)=6.79, p=.24\right)$ and noninteractive $\left(Q_{W}(15)=12.19, p=.66\right)$ effect sizes.

Similarly, we found no differences within $\left(Q_{W}(13)=11.65, p=.56 ; Q_{W}(7)=7.11, p=.42\right.$, respectively) or between $\left(Q_{b}(1)=.003, p=.95\right)$ studies measuring active $(d=.35, k=14, p$ $<.001,95 \% \mathrm{CI}=.21-.50)$ and passive $(d=.36, k=8, p<.001,95 \% \mathrm{CI}=.20-.53)$ forms of aggression. Thus, altering the degree to which participants interacted with or directly aggressed against a female failed to modify the effects of alcohol.

Assessment Method-An examination of the mean effect sizes for studies in which aggression was observed $(d=.38, k=10, p<.001,95 \% \mathrm{CI}=.21-.56)$ or reported $(d=.34$, $k=12, p<.001,95 \% \mathrm{CI}=.21-.48)$ revealed no between $\left(Q_{b}(1)=.12, p=.72\right)$ or within $\left(Q_{W}(9)=8.15, p=.52 ; \mathrm{Q}_{\mathrm{W}}(11)=10.66, p=.47\right.$, respectively) group differences. A similar relationship between alcohol and aggression emerged, regardless of assessment method.

Sample Type-Alcohol-aggression effect sizes for studies involving primarily college participants $(d=.43, k=11, p<.001,95 \% \mathrm{CI}=.28-.58)$ approached medium magnitude but were not significantly larger than effect sizes for studies of community samples $(d=.28$, $k=8, p<.001,95 \% \mathrm{CI}=.12-.44)$ as revealed by a formal test of homogeneity $\left(Q_{b}(1)=\right.$ $1.75, p=.19)$. Little variability was detected among college $\left(Q_{W}(10)=9.56, p=.48\right)$ and community $\left(Q_{W}(7)=6.18, p=.52\right)$ effect sizes.

Alcohol Dose-Homogeneity analyses $\left(Q_{b}(1)=.07, p=.79\right)$ revealed that administration of sufficient alcohol to achieve legal intoxication $(d=.37, k=11, p<.001,95 \% \mathrm{CI}=.21-$. $\left.54 ; Q_{W}(10)=10.02, p=.44\right)$ did not result in a stronger relationship between alcohol and 
aggression than observed among studies that administered smaller doses of alcohol ( $d=.34$, $\left.k=8, p<.001,95 \% \mathrm{CI}=.17-.51 ; Q_{W}(7)=5.94, p=.55\right)$.

\section{Discussion}

Pooling data from 2,566 participants in 22 studies that evaluated the effects of experimentally manipulated alcohol on male-to-female aggression revealed a small but significant effect of acute alcohol consumption on aggression at the aggregate level, indicating that participants who had received alcohol scored approximately one third of a standard deviation higher on measures of aggression than participants who had not received alcohol. Assignment to an alcohol or a control condition allows us to presume that drinking habits and individual differences (e.g., impulsivity, hostility) were equally distributed across groups and that any observed differences between groups were the result of the alcohol manipulation. Thus, the composite effect size represents the effect of acute alcohol consumption on male-to-female aggression, providing compelling support for the proximal, causal effects of alcohol (e.g., pharmacological; expectancy) in the perpetration of male-tofemale aggression.

The observed overall effect size was small, whereas prior meta-analyses yielded slightly larger effect sizes in the small-to-medium range. As discussed earlier, however, previous reviews evaluated the effects of proxies for problematic drinking, which may be confounded with personality characteristics associated with aggression (e.g., impulsivity) and are indicative of more pathological or severely aggressive individuals than assignment to an alcohol group under an experimental design. Therefore, we would expect the pure effect of acute alcohol consumption on male-to-female aggression to be smaller than proxy measures of problematic alcohol use (e.g., Foran \& O’Leary, 2008).

We detected no significant differences in the relationship between alcohol and aggression among SA and IPA studies. Analyses suggested a trend in which alcohol seemed to exert greater effects within the context of partner aggression than sexual aggression. It should be noted that moderator analyses were underpowered due to the small initial set of included studies as well as further reductions resulting from missing data on moderating variables. Although a greater number of investigations may provide adequate power to detect differences between the two types of male-to-female aggression, caution must be taken when making direct comparisons between constructs that are assessed through different paradigms. Thus, the current analyses indicate that alcohol is a comparable proximal risk factor for both SA and IPA, as operationalized and assessed through established paradigms, across identified studies in the existing literature.

The detection of comparable small-to-medium effects for both sexual and intimate partner aggression indicates that alcohol is an important, though insufficient, explanation for a subset of aggressive behavior. Indeed, many other proximally-relevant variables, such as individual and situational characteristics, have been shown to contribute to the risk of SA and IPA perpetration at the event-level. For example, one investigation found that the effect of alcohol on aggressive male-to-female verbalizations was stronger among participants with high, relative to low, dispositional aggression (Eckhardt \& Crane, 2008). An emerging 
perspective goes even further in positing that the presence of alcohol increases the risk of SA or IPA only among males already predisposed toward aggression (e.g., Abbey, 2011). A review of the studies included in the current meta-analysis revealed several examples of this type of interaction. For example, Eckhardt (2007) found that alcohol administration increased IPA only among previously violent husbands. Similarly, Abbey and colleagues (2009) determined that the effects of alcohol on SA were confined to participants who reported high dispositional levels of hostility. Other investigations have found that the relationship between alcohol and SA was mediated by participant sexual arousal (Davis et al., 2006), perceived female arousal (Davis et al., 2012), and perceived female enjoyment (Norris et al., 2002). Leonard and colleagues (Fals-Stewart, Leonard, \& Birchler, 2005) have explicated interactions between alcohol and dispositional or situational characteristics under a "multiple thresholds theory" in which alcohol may increase the severity but not frequency of aggression among males with the greatest predisposition toward aggressive responding (e.g., high dispositional hostility or a likelihood to perceive ambiguous sexual responses as consent or enjoyment). Similarly, alcohol may be sufficient to increase the frequency of lowlevel aggression among those moderately predisposed to aggressive responding and alcohol may be insufficient to elicit aggression from generally nonaggressive males. With few studies evaluating specific intervening variables, this level of specificity was beyond the capability of the current review but future meta-analyses may offer additional support for a multiple thresholds approach to interpreting the effects of alcohol on aggression.

Few alcohol administration studies of aggression have used high-risk samples consisting of those at the most severe end of the multiple thresholds model. In fact, the stringent selection criteria involved in alcohol administration studies may contribute to smaller effects of alcohol observed in laboratory experiments relative to naturalistic settings (e.g., Testa, VanZile-Tamsen, Livingston, \& Buddie, 2006). The small-to-medium effect reported in the current and previous reviews may not accurately represent the effects of alcohol among clinical samples at greater risk for aggression, such as violent offenders or substance abuse clients. Indeed, a small subset of early studies offer evidence to suggest that alcohol exerts significant effects on partner aggression among alcohol dependent husbands (Haber \& Jacob, 1997; Jacob \& Krahn, 1988; Jacob \& Leonard, 1988) and that these effects may be most evident among antisocial, alcohol dependent men (Jacob, Leonard, \& Haber, 2001). Thus, we encourage additional research using higher risk samples to increase ecological validity. Although ethical concerns have been raised, recent investigations suggest that alcohol administration studies of aggression do not increase subsequent use of alcohol (Pratt \& Davidson, 2005) or aggression (Parrott, Miller, \& Hudepohl, in press). Further, the benefits to be gained from studying those at greatest risk for future aggression warrant focused research that may result in advancing the effectiveness of prevention and intervention efforts.

The current composite effect sizes for general, sexual, and intimate partner aggression are consistent with the proximal effects models describing the role of acute alcohol intoxication in male-to-female aggression. As previously discussed, the psychopharmacological effects of alcohol are described as the causal mechanism under proximal effects models of disinhibition and attention allocation (e.g., Steele \& Josephs, 1990). Administration of alcohol in the laboratory is thought to impair the rapidity of higher order cognitive functions 
that are critical for optimal social functioning (Giancola et al., 2010). As a result, male participants who received alcohol may have been more likely than sober controls to engage in immediately gratifying behaviors, such as sexual or intimate partner aggression, possibly discounting prosocial behavioral options with the greatest long-term benefits, such as appropriate courting or moderate discussions of relationship problems.

Alternative theories attempt to explain the association between alcohol and aggression without relying upon the psychopharmacological effects of alcohol. Expectancy theories, for example, posit that participants who believe that the consumption of alcohol may partially or entirely exculpate them of responsibility for socially unacceptable aggressive interpersonal behavior may be prone to SA and IPA following alcohol consumption (e.g., Critchlow, 1983). The alcohol literature is not particularly supportive of expectancy theories (e.g., Quigley \& Leonard, 2006) and a recent meta-analysis demonstrated that placebo manipulations are generally not successful at activating expectancies or dissociating expectancy from pharmacological effects (Schlauch et al., 2010).

The current results are inconsistent with theoretical models that attribute all male-to-female aggression to societal influences (e.g., Dobash \& Dobash, 1979). A set of theoretical orientations, collectively referred to as feminist or neo-feminist models, contending that males aggress to gain power or exert control over females that are seen as subordinates by a larger patriarchal society that condones male-to-female aggression (Pence \& Paymar, 1993). Under this orientation, and consistent with expectancy theories, acute alcohol consumption is seen as an unacceptable excuse for, rather than a contributing cause of, aggression. The acknowledgement of alcohol's role is considered counterproductive for nonviolent cognitive and behavioral change. The current meta-analysis provides empirical, atheoretical support for a direct, causal relationship between alcohol and male-to-female aggression, suggesting that alcohol increases aggression under some circumstances for some men. By extension, theoretical approaches and the treatment programs that they inform may best serve victims by acknowledging and accommodating the observed variability in both the characteristics of perpetrators as well as precipitants to specific aggressive acts.

We detected homogeneity across all moderator categories of the collected effect sizes. The number of qualifying studies may have limited our ability to detect moderators that sufficiently explained variability in the alcohol-aggression relationship across studies. Thus we observed similar effect sizes across all specified categories of interaction, assessment method, sample type, and alcohol quantity. We found evidence of a potentially meaningful trend toward a stronger relationship between alcohol and aggression among college, as opposed to community, samples. It is possible that alcohol exerts greater effects upon college-age participants due to higher levels of impulsivity or associated sexual or aggressive behaviors that commonly occur within the drinking context of youths (Brown et al., 2008). Further comprehensive research may provide insight into which samples and experimental procedures yield the greatest and most accurate alcohol effects on aggression.

\section{Limitations and Future Directions}

The greatest limitation to the current review was the number of eligible studies identified in the existing aggression literature. The preponderance of aggression research involves same- 
sex pairings with only a minority addressing inter-gender aggression directly. We strongly encourage researchers to continue conducting additional, rigorous experimental evaluations of the effects of alcohol on male-to-female aggression. As stated, our composite effect size and null tests of moderation are likely an oversimplification of the complexity of the alcohol-aggression relationship predicated by an insufficient body of research. Despite the complexity of alcohol administration studies, including resistance from regulating bodies and practical complications associated with alcohol administration and participant compensation, carefully controlled experimental methods remain the gold standard for establishing a causal relationship between alcohol intoxication and adverse behavioral outcomes that cannot be determined through cross-sectional or event-level methods alone (Davis et al., 2014).

Nevertheless, it should be noted that the validity of laboratory aggression paradigms has been called into question (e.g., Tedeschi and Quigley, 1996). Chief among the concerns levied by proponents of reform is that laboratory aggression paradigms lack ecological validity in that participants are placed in an artificial situation and typically exposed to stimuli (e.g., insults from a simulated partner) and response options (e.g., subtract points from your partner's total) that fail to replicate those experienced in the real world (for discussion, see Testa, Crane, Quigley, Levitt, \& Leonard, 2014). Clearly, researchers cannot evaluate actual sexual or intimate partner physical aggression in the laboratory and must rely on proxy measures. Additionally, quantities of alcohol consumed in the lab are regulated, fail to represent the continuum of intoxication observed at home or in social situations that involve alcohol consumption, and may not reach the typical participant's threshold for disinhibition of aggression. Future experimental research should explore more ecologically valid experimental designs, such as interactive or home based virtual methods of assessing aggression and gauging alcohol consumption. Prospective daily diary and ecological momentary assessment strategies (e.g. Testa \& Derrick 2014) can also be integrated as supplemental components of larger experimental investigations and may represent a parallel pool of studies for additional reviews on this topic in the years to come.

\section{Conclusions}

Leading researchers and practitioners are in agreement that heavy, acute alcohol consumption is a contributing cause of male-to-female sexual and intimate partner aggression (e.g., Leonard, 2005; Abbey et al., in press). The current review provides the first comprehensive quantification of these specific effects across studies, suggesting that male participants who consume alcohol are significantly more aggressive toward females than those who do not $(d=.36)$ and that alcohol is a comparable proximal risk factor for both sexual $(d=.32)$ and intimate partner $(d=.45)$ aggression. Additional research will offer deeper insight into the multitude of individual and situational characteristics that interact with acute alcohol consumption to provide more reliable predictions of high risk individuals or scenarios and aid in the construction of optimal, individualized treatment plans for perpetrators of alcohol-involved aggression. Research involving clinical samples may result in stronger predictors of recidivism and more accurate risk assessment, thus reducing subsequent female victimization. A firm understanding of the precipitants of male-to-female aggression is paramount to reducing the frequency and severity of its occurrence.

Trauma Violence Abuse. Author manuscript; available in PMC 2017 December 01. 


\section{Acknowledgments}

This research was supported by grants from the National Institute on Alcohol Abuse and Alcoholism (T32-

AA007583, PI: Leonard; K23 AA021768, PI: Schlauch).

\section{References}

Abbey A. Alcohol's role in sexual violence perpetration: Theoretical explanations, existing evidence, and future directions. Drug and Alcohol Review. 2011; 30:481-489. [PubMed: 21896070]

*. Abbey A, Buck PO, Zawacki T, Saenz C. Alcohol's effects on perceptions of a potential date rape. Journal of Studies on Alcohol and Drugs. 2003; 64:669-677.

Abbey A, Jacques-Tiura A. Sesual assault perpetrators' tactics: Associations with their personal characteristics and aspects of the incident. Journal of Interpersonal Violence. 2011; 26:2866-2889. [PubMed: 21156685]

Abbey A, McAuslan P, Ross LT. Sexual assault perpetration by college men: the role of alcohol, misperception of sexual intent and sexual beliefs and experiences. Journal of Social and Clinical Psychology. 1998; 17:167-195.

*. Abbey A, Parkill MR, Jacques-Tiura AJ, Saenz C. Alcohol's role in men's use of coercion to obtain unprotected sex. Substance Use \& Misuse. 2009; 44:1329-1348. [PubMed: 19938921]

Abbey A, Wegner R, Woerner J, Pegram SE, Pierce J. Review of survey and experimental research that examine the relationship between alcohol consumption and men's sexual aggression perpetration. Trauma, Violence, \& Abuse. (in press).

Archer J. Sex differences in aggression between heterosexual partners: A meta-analytic review. Psychological Bulletin. 2000; 126:651-680. [PubMed: 10989615]

Archer J. Sex differences in aggression in real-world settings: A meta-analytic review. Review of General Psychology. 2004; 8:291-322.

Breiding MJ, Smith SG, Basile KC, Walters ML, Chen J, Merrick MT. Prevalence and characteristics of sexual violence, stalking, and intimate partner violence victimization-National Intimate Partner and Sexual Violence Survey, United States, 2011. MMWR: Surveillance Summaries. 2014; 63:118.

Brown S, McGue M, Maggs J, Schulenberg J, Hingson R, Murphy S. A developmental perspective on alcohol and youths 16 to 20 years of age. Pediatrics. 2008; 121:5290-5310.

Bushman, BJ. Effects of alcohol on human aggression: Validity of proposed explanations. In: Galanter, M., editor. Recent developments in alcoholism: Alcohol and violence. Vol. 13. New York: Plenum Press; 1997. p. 227-243.

Centers for Disease Control and Prevention, National Center for Injury Prevention and Control. Webbased Injury Statistics Query and Reporting System (WISQARS) [online]. [cited 2014 Nov 18]. Available from URL: http://www.cdc.gov/injury/wisqars

Clements K, Schumacher JA. Perceptual biases in social cognition as potential moderators of the relationship between alcohol and intimate partner violence: A review. Aggression and Violent Behavior. 2010; 15:357-368.

Coker AL, Davis KE, Arias I, Desai S, Sanderson M, Brandt HM, Smith PH. Physical and mental health effects of intimate partner violence for men and women. American Journal of Preventative Medicine. 2002; 23:260-268.

Critchlow B. Blaming the booze: The attribution of responsibility for drunken behavior. Personality and Social Psychology Bulletin. 1983; 9:451-473.

*. Davis KC. The influence of alcohol expectancies and intoxication on Men's aggressive unprotected sexual intentions. Experimental and Clinical Psychopharmacology. 2010; 18:418-428. [PubMed: 20939645]

*. Davis KC, Norris J, George WH, Martel J, Heiman JR. Men's likelihood of sexual aggression: The influence of alcohol, sexual arousal, and violent pornography. Aggressive Behavior. 2006; 32:581-589. 
Davis KC, Parrott DJ, George WH, Tharp AT, Hall GCN, Stappenbeck CA. Studying sexual aggression: A review of the evolution and validity of laboratory paradigms. Psychology of Violence. 2014; 4:462-476.

*. Davis KC, Schraufnagel TJ, Jacques-Tiura AJ, Norris J, George WH, Kiekel PA. Childhood sexual abuse and acute alcohol effects on men's sexual aggression intentions. Psychology of Violence. 2012; 2:179-193. [PubMed: 22754720]

Davison GC, Robins C, Johnson MK. Articulated thoughts during simulated situations: A paradigm for studying cognition in emotion and behavior. Cognitive Therapy and Research. 1983; 7:17-39.

Dobash, R.; Dobash, R. Violence against wives. NY: The Free Press; 1979.

Eagly A, Steffen VJ. Gender and aggressive behavior: A meta-analytic review of the social psychological literature. Psychological Bulletin. 1986; 100:309-330. [PubMed: 3797558]

*. Eckhardt CI. Effects of alcohol intoxication on anger experience and expression among partner assaultive men. Journal of Consulting and Clinical Psychology. 2007; 75:61-71. [PubMed: 17295564]

*. Eckhardt C, Crane C. Effects of alcohol intoxication and aggressivity on aggressive verbalizations during anger arousal. Aggressive Behavior. 2008; 34:428-436. [PubMed: 18307248]

Exum ML. Alcohol and aggression: An integration of findings from experimental studies. Journal of Criminal Justice. 2006; 34:131-145.

Fals-Stewart W, Leonard KE, Birchler GR. The occurrence of male-to-female intimate partner violence on days of men's drinking: the moderating effects of antisocial personality disorder. Journal of Consulting and Clinical Psychology. 2005; 73:239-248. [PubMed: 15796631]

Ferrer V, Bosch E, Garcia E, Manassero MA, Gili M. Meta-analytic study of differential characteristics between batterers and non-batterers: The case of psychopathology and consumption of alcohol and drugs. Psykhe. 2004; 13:141-156.

Foran HM, O’Leary KD. Alcohol and intimate partner violence: A meta-analytic review. Clinical Psychology Review. 2008; 28:1222-1234. [PubMed: 18550239]

Giancola PR, Josephs RA, Parrott DJ, Duke AA. Alcohol myopia revisited clarifying aggression and other acts of disinhibition through a distorted lens. Perspectives on Psychological Science. 2010; 5:265-278. [PubMed: 26162159]

*. Gross AM, Bennett T, Sloan L, Marx BP, Juergens J. The impact of alcohol and alcohol expectancies on male perception of female sexual arousal in a date rape analog. Experimental and Clinical Psychopharmacology. 2001; 9:380-388. [PubMed: 11764014]

Haber JR, Jacob T. Marital interactions of male versus female alcoholics. Family Process. 1997; 36:385-402. [PubMed: 9543660]

Hedges, L.; Olkin, I. Statistical methods for meta-analysis. Orlando, FL: Academic Press; 1985.

Heyman RE, Weiss RL, Eddy JM. Marital interaction coding system: Revision and empirical evaluation. Behaviour Research and Therapy. 1995; 33:737-746. [PubMed: 7654167]

Ito TA, Miller N, Pollock VE. Alcohol and aggression: A meta-analysis on the moderating effects of inhibitory cues, triggering events, and self-focused attention. Psychological Bulletin. 1996; 120:60-82. [PubMed: 8711017]

Jacob T, Krahn GL. Marital interactions of alcoholic couples: Comparison with depressed and nondistressed couples. Journal of Consulting and Clinical Psychology. 1988; 56:73-79. [PubMed: 3346452]

Jacob T, Leonard KE. Alcoholic-spouse interaction as a function of alcoholism subtype and alcohol consumption interaction. Journal of Abnormal Psychology. 1988; 97:231-237. [PubMed: 3385076]

Jacob T, Leonard KE, Haber J. Family interactions of alcoholics as related to alcoholism type and drinking condition. Alcoholism: Clinical and Experimental Research. 2001; 25:835-843.

*. Johnson JD, Noel NE, Sutter-Hernandez J. Alcohol and male acceptance of sexual aggression: The role of perceptual ambiguity. Journal of Applied Social Psychology. 2000; 30:1186-1200.

Leonard KE. Alcohol and intimate partner violence: when can we say that heavy drinking is a contributing cause of violence? Addiction. 2005; 100:422-425. [PubMed: 15784050] 
*. Leonard KE, Roberts LJ. The effects of alcohol on the marital interactions of aggressive and nonaggressive husbands and their wives. Journal of Abnormal Psychology. 1998; 107:602-615. [PubMed: 9830248]

Lipsey, M.; Wilson, D. Practical Meta-Analysis. Thousand Oaks, CA: Sage Publications, Inc; 2001.

Lipsey, MW.; Wilson, DB.; Cohen, MA.; Derzon, JH. Is there a causal relationship between alcohol use and violence? A synthesis of evidence. In: Galanter, M., editor. Recent developments in alcoholism: Alcohol and violence. Vol. 13. New York: Plenum Press; 1997. p. 245-282.

*. Martell, JF. Doctoral dissertation. University of Washington; 2003. The effects of alcohol, executive cognitive function, individual differences, and contextual variables on college men's perceptions of unwanted sexual advances.

*. Marx BP, Gross AM, Juergens JP. The effects of alcohol consumption and expectancies in an experimental data rape analogue. Journal of Psychopathology and Behavioral Assessment. 1997; 19:281-302.

*. Miller, CA.; Parrott, DJ. Doctoral Dissertation. Georgia State University; 2013. When do men perceive that 'no' means 'yes'?: Effects of alcohol and men's expectancies of intoxicated women's sexual desire and vulnerability on sexual aggression.

*. Noel NE, Maisto SA, Johnson JD, Jackson LA. The effects of alcohol and cue salience on young men's acceptance of sexual aggression. Addictive Behaviors. 2009; 34:386-394. [PubMed: 19108956]

*. Norlander T, Nordmarker A, Archer T. Effects of alcohol and frustration on experimental graffiti. Scandinavian Journal of Psychology. 1998; 39:201-207. [PubMed: 9883098]

*. Norris J, Davis KC, George WH, Martell J, Heiman JR. Alcohol's direct and indirect effects on men's self-reported sexual aggression likelihood. Journal of Studies on Alcohol and Drugs. 2002; 63:688-695.

*. Norris J, Kerr KK. Alcohol and violent pornography: Responses to permissive and nonpermissive cues. Journal of Studies on Alcohol and Drugs. 1993; (Supplement No. 11):118-127.

Orwin RG. A fail safe N for effect size in meta-analysis. Journal of Educational Statistics. 1983; 8:157-159.

Parrott DJ, Miller CA, Hudepohl AD. Immediate and short-term reactions to participation in laboratory aggression research. Psychology of Violence. (in press).

Pence, E.; Paymar, M. Education groups for men who batter: The Duluth Model. New York: Springer; 1993.

Perkins H. Surveying the damage: A review of research on consequences of alcohol misuse in college populations. Journal of Studies on Alcohol and Drugs. 2002; 14:91-100.

Pratt WM, Davidson D. Does participation in an alcohol administration study increase risk for excessive drinking? Alcohol. 2005; 37:135-141. [PubMed: 16713501]

Quigley BM, Leonard KE. Alcohol expectancies and intoxicated aggression. Aggression and Violent Behavior. 2006; 11:484-496.

*. Quinones, B. Doctoral dissertation. University of South Florida; 1998. The effects of alcohol on men's imposition of sexually explicit material on a female confederate: The role of "suggestive" and "nonsuggestive" cues.

*. Richardson D. The effects of alcohol on male aggression toward female targets. Motivation and Emotion. 1981; 5:333-344.

Roizen J. Epidemiological issues in alcohol-related violence. Recent Developments in Alcohol. 1993; 13:7-37.

Rosenthal, R. Meta-analytic procedures for social research. Vol. 6. Thousand Oaks, CA: Sage Publications, Inc; 1991.

Saltzman, L.; Fanslow, J.; McMahon, P., et al. Intimate partner violence surveillance: uniform definitions and recommended data elements, version 1.0. Atlanta, GA: Centers for Disease Control and Prevention, National Center for Injury Prevention and Control; 2002.

*. Samp JA, Monahan JL. Alcohol-influenced nonverbal behaviors during discussions about a relationship problem. Journal of Nonverbal Behavior. 2009; 33:193-211. 
Schlauch RC, Waesch M, Riccardi C, Donohue K, Blagg C, Christensen R, Lang AR. A meta-analysis of the effectiveness of placebo manipulations in alcohl-challenge studies. Psychology of Addictive Behaviors. 2010; 24:239-253. [PubMed: 20565150]

*. Stappenbeck CA, Fromme K. The effects of alcohol, emotion regulation, and emotional arousal on the dating aggression intentions of men and women. Psychology of Addictive Behaviors. 2014; 28:10-19. [PubMed: 23586449]

Steele CM, Josephs RA. Alcohol myopia: Its prized and dangerous effects. American Psychologist. 1990; 45:921-933. [PubMed: 2221564]

Stith SM, Smith DB, Penn CE, Ward DB, Tritt D. Intimate partner physical abuse perpetration and victimization risk factors: A meta-analytic review. Aggression and Violent Behavior. 2004; 10:6598.

Taylor SP. Aggressive behavior and physiological arousal as a function of provocation and the tendency to inhibit aggression. Journal of Personality. 1967; 35:297-310. [PubMed: 6059850]

Taylor SP, Leonard KE. Alcohol and human physical aggression. Aggression: Theoretical and Empirical Reviews. 1983; 2:77-101.

Tedeschi JT, Quigley BM. Limitations of laboratory paradigms for studying aggression. Aggression and Violent Behavior. 1996; 1:163-177.

Testa M. The impact of men's alcohol consumption on perpetration of sexual aggression. Clinical Psychology Review. 2002; 22:1239-1263. [PubMed: 12436812]

*. Testa M, Crane CA, Quigley BM, Levitt A, Leonard KE. Effects of administered alcohol on intimate partner interactions in a conflict resolution paradigm. Journal of Studies on Alcohol and Drugs. 2014; 75:249-258. [PubMed: 24650819]

Testa M, Derrick JL. A daily process examination of the temporal association between alcohol use and verbal and physical aggression in community couples. Psychology of Addictive Behaviors. 2014; 28:127-138. [PubMed: 24341618]

Testa M, VanZile-Tamsen C, Livingston JA, Buddie AM. The role of women's alcohol consumption in managing sexual intimacy and sexual safety motives. Journal of Studies on Alcohol and Drugs. 2006; 67:665-674. 

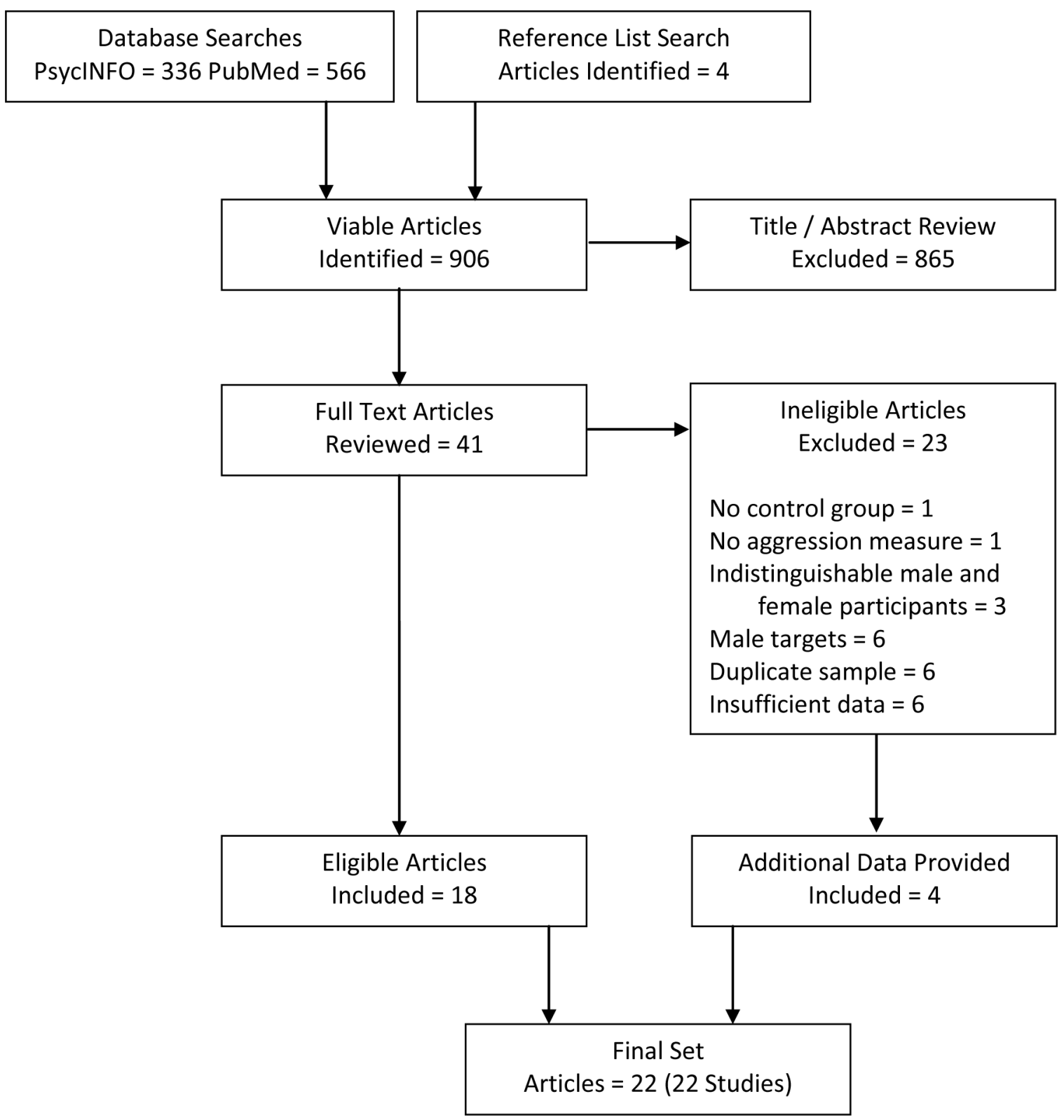

Figure.

The selection and review process that resulted in 22 articles for inclusion in the current meta-analysis. 


\section{Table 1}

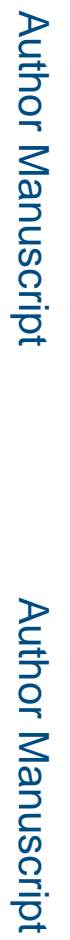

Plot of Unadjusted Study Effect Sizes.

\begin{tabular}{rl}
\hline$\underline{\text { Stem }}$ & Leaf \\
-.0 & 44 \\
.0 & 00,43 \\
.1 & $19,43,44,70$ \\
.2 & 22 \\
.3 & 24,29 \\
.4 & $03,65,74,91,95$ \\
.5 & 50,97 \\
.6 & $10,24,29$ \\
.7 & 45,81 \\
\hline
\end{tabular}

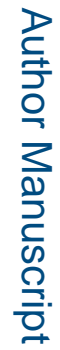

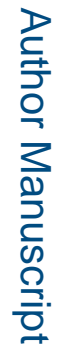

Trauma Violence Abuse. Author manuscript; available in PMC 2017 December 01. 
